\title{
Sensor-based Remote Temperature and Humidity Monitoring Device Embedded in Wheelchair Cushion
}

\author{
Yu-Sheng Yang, ${ }^{1}$ Cheng-Tang Pan, ${ }^{2}$ and Wen-Hsien $\mathrm{Ho}^{3,4 *}$ \\ ${ }^{1}$ Department of Occupational Therapy, Kaohsiung Medical University, \\ 100, Shih-Chuan 1st Road, Kaohsiung 80708, Taiwan, R.O.C. \\ ${ }^{2}$ Department of Mechanical and Electromechanical Engineering, National Sun Yat-sen University, \\ 70, Lienhai Road, Kaohsiung 80424, Taiwan, R.O.C. \\ ${ }^{3}$ Department of Healthcare Administration and Medical Informatics, Kaohsiung Medical University, \\ 100, Shih-Chuan 1st Road, Kaohsiung 80708, Taiwan, R.O.C. \\ ${ }^{4}$ Department of Medical Research, Kaohsiung Medical University Hospital, \\ 100, Shih-Chuan 1st Road, Kaohsiung 80708, Taiwan, R.O.C.
}

(Received May 15, 2017; accepted February 16, 2018)

Keywords: pressure ulcers, wireless technology, temperature

Increases in skin temperature and accumulation of moisture during prolonged sitting are risk factors for pressure ulcers. Therefore, a method of assessing temperature and moisture between the body and support surfaces is needed. The purpose of this study was to develop and evaluate a prototype for a wheelchair cushion equipped with remotely operated sensors to enable realtime monitoring of temperature and humidity by the wheelchair user. Twenty-six wheelchairbound patients gave informed consent to participate in this study. Participants were randomly assigned to sit naturally on either a hybrid foam-fluid cushion or an air-filled rubber cushion. During $2 \mathrm{~h}$ of sitting, a digital sensor was used to obtain bilateral temperature and humidity measurements at the ischial tuberosities and thighs. Temperature and humidity data collected by the sensors were processed with an Arduino Uno microcontroller. A Zigbee transceiver module mounted on the carrier board also enabled wireless transmission of the data to monitor the workstation. Experiments showed that this wireless, remotely operated monitoring system accurately detected changes in skin temperature and humidity, which were calculated as described in the literature. These vital signs could then be transmitted wirelessly to an online database for future access by other caregivers. The proposed device is a useful precautionary measure for ensuring the adequate monitoring of patients required to sit in wheelchairs for prolonged periods.

\section{Introduction}

Pressure ulcers, also known as decubitus ulcers or bedsores, are caused by pressure, shear force, and/or friction. These injuries to the skin and/or underlying tissue usually occur over a bony prominence. Pressure ulcers are usually localized injuries but can be life threatening if left untreated. They tend to affect people with health conditions that cause difficulty in moving *Corresponding author: e-mail: whho@kmu.edu.tw http://dx.doi.org/10.18494/SAM.2018.1872 
or require prolonged lying or sitting. Therefore, protection of soft tissue over the buttock areas is a vital concern to individuals who are unable to reposition themselves while confined to a bed or wheelchair. Because pressure is the key risk factor for pressure ulcers, pressure-reducing cushions are a commonly prescribed prophylactic against the development of pressure ulcers in wheelchair users. ${ }^{(1-4)}$

In addition to pressure, two other important contributing factors in the development of pressure ulcers are shear forces and microclimate conditions. ${ }^{(5-7)}$ In patients confined to wheelchairs or beds, the microclimate refers to the local temperature, humidity, and moisture conditions that affect the skin, particularly in bony prominences or other weight-bearing areas that interface with wheelchairs, beds, or clothing. Some studies show that a high skin temperature contributes to increased tissue metabolism and oxygen consumption. Thus, in addition to having a high risk of pressure ulcers, patients with compromised tissue also have higher than normal oxygen requirements. Thus, any combined increase in temperature and pressure may promote ulcer formation by reducing tissue viability. ${ }^{(7-9)}$

Another key extrinsic factor in pressure ulcer development is moisture. ${ }^{(10,11)}$ Potential sources of moisture that can degrade the skin include perspiration, urine, feces, and fistula or wound drainage. The presence of moisture-associated skin damage may increase pressure ulcer risk. A previous study demonstrated the detrimental effects of increased moisture adjacent to the skin by testing the tensile strength of excised skin specimens in a controlled humidity environment. The experiments showed that increasing the relative humidity from 10 to $98 \%$ resulted in a $75 \%$ decrease in tensile strength. ${ }^{(12)}$ Skin with such reduced strength is prone to mechanical damage from shear stress or abrasion. Therefore, the accumulation of moisture between skin surfaces and seat cushions is a potential contributor to pressure ulcer development.

To minimize the negative effects of temperature, pressure, and moisture on wheelchair users, in this study, we developed a prototype wheelchair cushion equipped with temperature and humidity sensors and a wireless remote connection to enable real-time monitoring. One potential application of the proposed temperature and humidity monitoring system is to improve healthcare quality in wheelchair-bound and bed-bound nursing home residents and to reduce the workload of caregivers.

\section{Materials and Methods}

\subsection{Data acquisition unit}

A parallel analogue-to-digital converter (ADC) module was used for the real-time acquisition and monitoring of temperature and relative humidity ( $\mathrm{RH})$. An Arduino Uno Rev3 board was selected for use in data sampling owing to its well-established reliability and stability. The Arduino Uno Rev3 board used in this study had 6 ADC channels with 10-bit resolution and $9600 \mathrm{~Hz}$ sampling frequency per channel. Figure 1 shows the Zigbee wireless transceiver module mounted on the Arduino board. The Zigbee module was selected owing to its well-established effectiveness in low-data-rate applications, low power consumption, and low cost. To simplify the implementation (configuration and initialization) of the device and to 


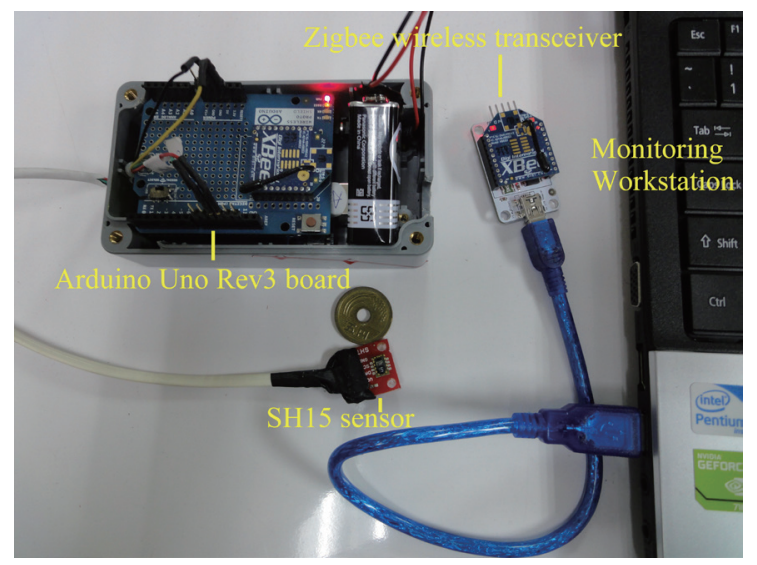

Fig. 1. (Color online) Prototype temperature and humidity monitoring system and sensor.

simplify data management (real-time data storage and offline analysis), a user-friendly graphical user interface (GUI) was developed in the Visual Basic Integrated Development Environment (Microsoft Co., Redmond, WA, USA). The first line of the GUI indicated the subject node attempting to join a network, and the second and third lines indicated the temperature and humidity readings. Notably, the measurement system was powered by a standard universal serial bus (USB) port or a battery pack to increase portability and to avoid errors when connecting the power supply to the sensors.

\subsection{Sensor}

Figure 1 shows the digital humidity and temperature sensor (Model SH15, Sensirion AG, Staefa, Switzerland) used to detect RH and temperature at the body-seat interface. The SH15 digital humidity and temperature sensor (dimensions: $2 \times 2 \times 0.25 \mathrm{~cm}^{3}$ ) contains a capacitivetype humidity sensor and a band-gap temperature sensor, and integrates the two sensors and readout circuitry on one single chip. This SH15 sensor is factory-calibrated and the calibration data are stored in on-chip nonvolatile memory. The temperature measurement range was -40 to $123.8^{\circ} \mathrm{C}$. The accuracy reported by the manufacturer when measuring temperatures at $25^{\circ} \mathrm{C}$ is $\pm 0.4{ }^{\circ} \mathrm{C}$. The RH measurement range was $0-100 \%$ RH with an accuracy within $\pm 2 \%$ between 0 and $90 \% \mathrm{RH}$. Response times for temperature and $\mathrm{RH}$ readings were 5 and $8 \mathrm{~s}$, respectively.

\subsection{Protocols for sitting experiments}

To evaluate the capability of the proposed remote system for detecting temperature and $\mathrm{RH}$ in a cushion user, bilateral measurements of skin surface temperature and RH were obtained at the ischial tuberosities and thighs. Each SH15 sensor was attached to the skin with a single strip of surgical tape. A computer randomization method was then used to assign each participant to one of the two cushion types. Since frequent postural movements could reduce temperature 
and moisture during sitting, participants were asked to perform $2 \mathrm{~h}$ of uninterrupted sitting with the buttocks and thighs remaining in constant contact with the seat cushion. Average temperature and $\mathrm{RH}$ at the ischial tuberosities and thighs over a 30 -s period were obtained before the participant sat on the cushion and then at 15-min intervals during the $2 \mathrm{~h}$ of sitting. As human body temperatures would not be expected to vary abruptly over short periods $(<1 \mathrm{~s})$, the sampling frequency of the measurement system was set at $1 \mathrm{~Hz} /$ sensor.

Two commercially available pressure-relief wheelchair cushions were used in this study: a dual compartment air-filled rubber cushion (ROHO low-profile cushion, ROHO Inc., Belleville, IL, USA; dimensions, $41 \times 41 \times 5 \mathrm{~cm}^{3}$ ) and a foam-fluid hybrid cushion incorporating a top layer of viscous fluid and a bottom layer of foam (Model HS-017, Hueishen Enter. Corp., Pingtung, Taiwan; dimensions, $40 \times 40 \times 7 \mathrm{~cm}^{3}$ ).

\subsection{Participants}

This study was approved by the Institutional Review Board of Kaohsiung Medical University Hospital. Before the experiment, written informed consent was obtained from all 26 participants. Patients were excluded if their medications or medical conditions affected body temperature (e.g., fever). Age range was 20 to 26 years, and BMI range was 18.33 to 28.09 $\mathrm{kg} \cdot \mathrm{m}^{-2}$. This study was a randomized controlled trial. All participants had an equal chance of being assigned to either one of two cushions. A randomized controlled trial was conducted because taking repeated measurements from subjects over time would more likely be affected by heat and humidity accumulation between the body and seat during long periods of sitting regardless of the type of cushion used. The experimental protocol required each participant to sit naturally for $2 \mathrm{~h}$ without lifting their buttocks and thighs from the assigned seat cushion. For each participant, the experiment was conducted within one day. To exclude the effects of different garment materials on sensor readings, all subjects wore similar short cotton pants during each experiment. All measurements were obtained in the same research room. During the experimental period, an additional SH15 sensor in the research room recorded an ambient temperature range of $24.5-25.5^{\circ} \mathrm{C}$ and an ambient $\mathrm{RH}$ range of $45-55 \%$.

\subsection{Data analysis}

Although its low power limits its transmission range, according to the manufacturer's datasheet, the Zigbee wireless module can transmit data up to $50 \mathrm{~m}$ with a clear line of sight. Therefore, to verify if the device can deliver its function under diverse environmental conditions, such as a nurses' station, a real-time monitoring station was set up in another room at a distance of $20 \mathrm{~m}$ from the research room. Temperature and RH data from the participants at the research room were transmitted to a real-time monitoring workstation just $20 \mathrm{~m}$ away and automatically stored on its hard disk drive.

The parameters used to analyze the effects of cushion properties included average temperature and RH (30-s epochs) obtained at each 15-min interval. Descriptive statistics were used to describe the basic features of skin temperature and $\mathrm{RH}$ at each time interval. 
Afterward, Pearson's correlation was used to determine the degree of correlation between the left-side and right-side data on ischial tuberosities and thighs. One-way analysis of variance was used to compare changes in skin temperature and RH between the two seat cushions over the 2-h sitting period. An alpha level of 0.05 was considered statistically significant. Statistical analyses were performed using IBM SPSS Statistics for Windows, Version 21.0 (IBM Corp, Armonk, NY).

\section{Results}

The proposed wireless remote temperature and $\mathrm{RH}$ monitoring device was tested in two separate rooms at a medical university hospital. The GUI of the coordinator set for the monitoring station was connected to the coordinator computer via a USB serial cable. Whenever the coordinator of the microcontroller unit received a temperature and $\mathrm{RH}$ reading, it sent a message to the coordinator computer. Clicking the 'Link start' button caused the GUI to read and chart the temperature and RH data for a particular subject on a given date. Once the message was received, the GUI converted the inputs to actual temperature and RH.

The operator of the monitoring device could assign an identity number to a patient. The identity number and name of the patient were then stored in a local folder in the database. The temperature record was saved in a text file, and the data were formatted as "Time-RHTemperature".

The wireless remote monitoring system was used to compare temperature and humidity data between two different cushions (13 participants for each cushion). Because the correlations between the right and left parameters were statistically significant $(p<0.01)$, representative values were obtained by averaging the values for the right and left sides. Figures 2(a) and 2(b) show time series plots of body temperature and $\mathrm{RH}$ measured at the ischial tuberosities

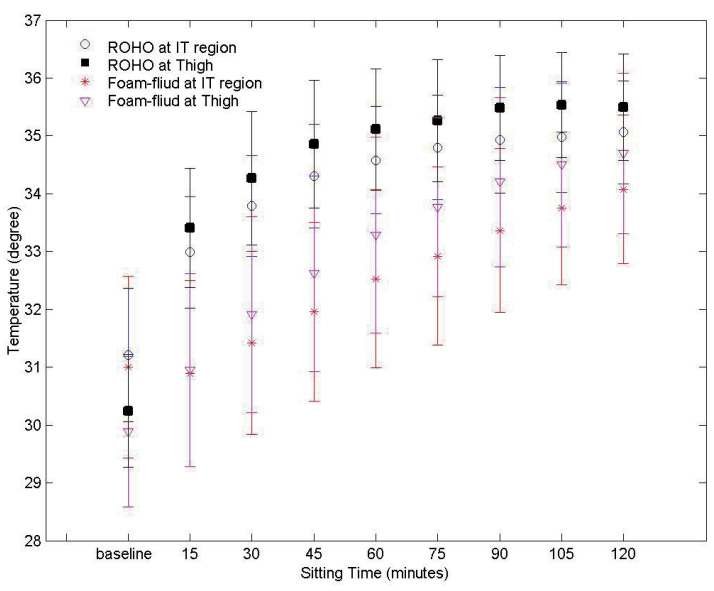

(a)

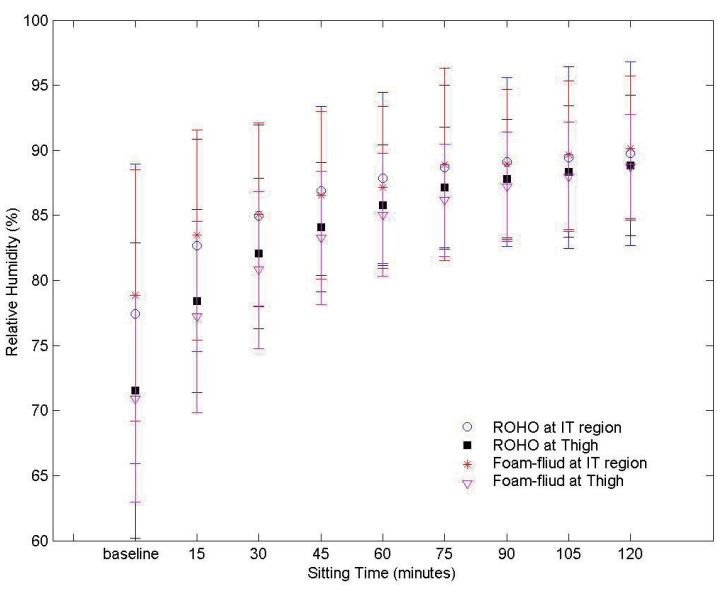

(b)

Fig. 2. (Color online) (a) Average temperature change and (b) average relative humidity in ischial tuberosity (IT) regions and in thighs during 2-h sitting period: comparison between air-filled (ROHO) and foam-fluid cushions. 
and thighs. The figures show that, after $2 \mathrm{~h}$ of unrelieved sitting, the two seat cushion types significantly differed in skin temperature measurements obtained at the ischial tuberosities ( $p$ $<0.01)$ and at the thighs $(p<0.01)$. Over time, the foam-fluid hybrid cushion had significantly lower skin temperature measurements than the ROHO cushion. However, the two cushion types did not significantly differ in RH measured in the thigh region or in ischial tuberosities. Regardless of cushion type, $\mathrm{RH}$ tended to increase over time.

\section{Discussion}

The proposed wireless remote temperature and humidity monitoring device accurately measured the temperature and RH of the participants in this study. The experiments also showed that the portable temperature and RH monitoring device was capable of accurate wireless transmission of data to the main station for monitoring. Measurements of body temperature and $\mathrm{RH}$ could then be stored in an online database that allowed caregivers to access the database from any location.

Compared with the ROHO cushion, the foam-fluid hybrid cushion resulted in lower average body temperature measurements in both the ischial tuberosities and thighs. However, the average RH did not differ significantly between the two cushions. The best explanation for this difference, which has also been observed in previous studies, ${ }^{(13)}$ is the different thermal conductivities of the materials used to fabricate the cushions. That is, because viscous fluids have a higher heat capacity than rubber, the foam-fluid hybrid cushion resulted in lower skin temperatures than the $\mathrm{ROHO}$ cushion after $2 \mathrm{~h}$ of sitting. On the other hand, each cushion was covered with a nonporous material such as rubber or viscous fluid padding. Therefore, the accumulation of moisture during $2 \mathrm{~h}$ of sitting would be expected to cause high humidity at the body-seat interface.

In both the ischial tuberosities and thigh region, the sensors accurately detected changes in body temperature and RH. Additionally, measurements on the right and left sides were very similar, which indicated that only one side of the thigh region would be sufficient for the accurate measurement of body temperature and RH. Future work can evaluate the use of sensors placed in small recesses cut into the cushion foam or covers. Sensors should be positioned to obtain measurements in the thigh region, which would not cause discomfort and may even be imperceptible since the thigh region has more fat and muscle than the ischial tuberosities.

The target populations using this device are those who sit for prolonged times in wheelchairs. Although monitoring once every $15 \mathrm{~min}$ is sufficient to detect adverse sedentary behavior because pressure relief in the form of periodic weight shifting must be done every 15-20 min, the proposed device can support data collection over shorter time-periods (e.g., every 5 min) to help record any other problems. The device offers an added precautionary measure to determine the current condition of a person. If the system detects a fall, it delivers alerts to the supporting people or healthcare organizations to summon first aid as soon as possible. Caregivers or nursing home staff can now know their patients' current status instantly and reduce their nursing workload. 


\section{Conclusions}

The wireless remote temperature and humidity monitoring device proposed in this study uses sensors to measure the effects of prolonged sitting. The sensor-based measurement system enabled the remote monitoring of body temperature and humidity at the user-seat interface. The Zigbee protocol was used for the wireless transmission of temperature and $\mathrm{RH}$ data to a monitoring station connected to a wireless mesh network. The user-seat interface coordinated the processes of monitoring, transmitting, alerting, and recording of temperature and RH. The experimental results indicate that the remote monitoring system can reliably monitor temperature and $\mathrm{RH}$ changes at this user-seat interface and that the device is unaffected by pressure imposed on sensors during normal sitting.

This proposed wireless remote monitoring device utilizes both real-time and stored information to accurately deliver an alarm according to the patient's current activity. If a patient falls, an alarm is delivered to supporting people to reduce the latency in providing medical attention to the patient. Through the use of existing low-cost technologies, this monitoring system could deliver significantly improved overall quality of patient care.

In future studies, the remote temperature and $\mathrm{RH}$ monitoring device will be further developed into a low-cost product with plug-and-play features that enable use by an untrained caregiver with minimal assistance. The software interface for users will also be improved, and the downloading and installation procedures will be simplified. Our future work will also evaluate the use of other wireless network protocols, e.g., Bluetooth, for remote monitoring. Because most smartphones now support Bluetooth, caregivers can benefit from technologies that enable them to monitor patients using smartphones.

\section{Acknowledgments}

This work was supported by the National Sun Yat-Sen University-Kaohsiung Medical University (NSYSU-KMU) joint research project (NSYSUKMU 103-I007), the Ministry of Science and Technology, Taiwan, under grant No. MOST 105-2622-E-037-005-CC3, and MOST 106-2218-E-327-001, and the "Intelligent Manufacturing Research Center" (iMRC) from the Featured Areas Research Center Program within the framework of the Higher Education Sprout Project by the Ministry of Education (MOE) in Taiwan.

\section{References}

1 D. Brienza, S. Kelsey, P. Karg, A. Allegretti, M. Olson, M. Schmeler, J. Zanca, M. J. Geyer, M. Kusturiss, and M. Holm: J Am. Geriatr. Soc. 58 (2010) 2308.

2 D. M. Brienza, P. E. Karg, M. J. Geyer, S. Kelsey, and E. Trefler: Arch. Phys. Med. Rehabil. 82 (2001) 529.

3 M. J. Geyer, D. M. Brienza, P. Karg, E. Trefler, and S. Kelsey: Adv. Skin Wound Care 14 (2001) 120.

4 L. Stockton and S. Rithalia: J. Tissue Viability 18 (2009) 28.

5 W. Sae-Sia, D. D. Wipke-Tevis, and D. A. Williams: Adv. Skin Wound Care 88 (2007) 1673.

6 V. Schubert and B. Fagrell: Scand. J. Rehabil. Med. 23 (1991) 33.

7 V. Schubert, L. Perbeck, and P. A. Schubert: Clin. Physiol. 14 (1994) 1.

8 S. Patel, C. F. Knapp, J. C. Donofrio, and R. Salcido: J. Rehabil. Res. Dev. 36 (1999) 189.

9 S. Sprigle, M. Linden, D. McKenna, K. Davis, and B. Riordan: Adv. Skin Wound Care 14 (2001) 133. 
10 B. Braden and N. Bergstrom: Rehabil. Nurs. 12 (1987) 8.

11 J. E. Grey, K. G. Harding, and S. Enoch: Br. Med. J. 332 (2006) 472.

12 R. H. Wildnauer, J. W. Bothwell, and A. B. Douglass: J. Invest. Dermatol. 56 (1971) 72.

13 T. W. Hsu, S. Y. Yang, J. T. Liu, C. T. Pan, and Y. S. Yang: Assist. Technol. 30 (2018) 1.

\section{About the Authors}

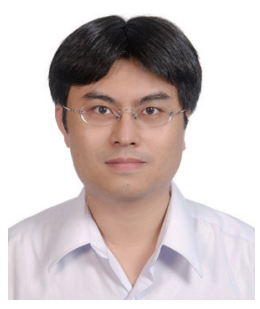

Yu-Sheng Yang graduated from the Ph.D. program, School of Rehabilitation Science and Technology, University of Pittsburgh, USA, in 2005. He received his MA degree from the Department of Occupational Therapy, New York University, USA, in 2000. He is currently an associate professor at the Department of Occupational Therapy, Kaohsiung Medical University, Kaohsiung, Taiwan. He also serves as an adjunct occupational therapist at Chung-Ho Memorial Hospital, Kaohsiung, Taiwan. His main research interests include assistive technology, wheelchair biomechanics, and motion analysis in human movement.

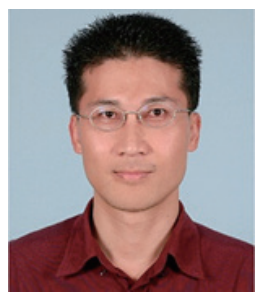

Cheng-Tang Pan received his master's and doctoral engineering degrees in 1993 and 1998, respectively, from the Power Mechanical Engineering Department of National Tsing Hua University, Hsinchu, Taiwan. He was a researcher in the field of laser machining polymers in TU Berlin (IWF), Germany, from 1997 to 1998 and a researcher in the MEMS Division in MIRL/ITRI, Hsinchu, Taiwan from 1998 to 2003. He joined National Sun Yat-Sen University, Kaohsiung, Taiwan, as an assistant professor in 2003, then earned his associate professor and full professor ranks in 2005 and 2008, respectively. He won the Outstanding Professor Award (2009-2013) from National Sun Yat-Sen University. From June 2009 to June 2010, he was a visiting professor at the Department of Mechanical Engineering in UC Berkeley. His current research interests focus on MEMS, nanofabrication, microscale energy, and LIGA process.

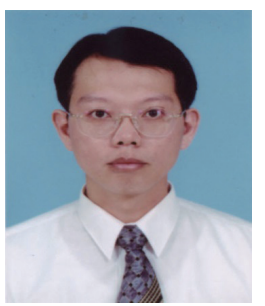

Wen-Hsien Ho received his B.S. degree in industrial and information management from National Cheng-Kung University, M.S. degree in mechanical and automation engineering from National Kaohsiung First University of Science and Technology, Taiwan, and Ph.D. degree in engineering science and technology from National Kaohsiung First University of Science and Technology, Taiwan, in 1998, 2002, and 2006, respectively. He is currently a professor in the Department of Healthcare Administration and Medical Informatics, Kaohsiung Medical University, Taiwan. His research interests include intelligent systems and control, computational intelligence and methods, and quality engineering. 